\title{
Mast Cells in Irritable Bowel Syndrome and Ulcerative Colitis: Function Not Numbers Is What Makes All the Difference
}

\author{
Theoharis C. Theoharides
}

Received: 5 December 2013/Accepted: 5 December 2013/Published online: 21 January 2014

(C) Springer Science+Business Media New York 2014

\section{Background and Significance of Issues and Controversies}

Data obtained from animal and human studies support the existence of mast cells (MC) in the gastrointestinal (GI) tract, where they are considered important for the control of luminal parasites and possibly other microorganisms. MC are also implicated in food allergies, GI inflammation, and functional GI diseases, including irritable bowel syndrome (IBS) [1]. MC density may be increased in inflammatory bowel disease (IBD) and IBS, although such studies have yielded variable and sometimes contradictory results, likely due to methodological problems such as sampling issues, poorly characterized MC identification methodology, and lack of adequate documentation of disease correlates. There is no question that $\mathrm{MC}$ numbers are likely to be easier to identify and count in lesional areas as they may be present in IBD.

\section{T. C. Theoharides $(\square)$}

Molecular Immunopharmacology and Drug Discovery

Laboratory, Department of Integrative Physiology and

Pathobiology, Tufts University School of Medicine,

136 Harrison Avenue, Boston, MA 02111, USA

e-mail: theoharis.theoharides@tufts.edu

T. C. Theoharides

Sackler School of Graduate Biomedical Sciences, Tufts

University, Boston, MA 02111, USA

T. C. Theoharides

Department of Internal Medicine, Tufts University School of

Medicine and Tufts Medical Center, Boston, MA 02111, USA

T. C. Theoharides

Department of Psychiatry, Tufts University School of Medicine and Tufts Medical Center, Boston, MA 02111, USA
MC density in the terminal ileum had previously been reported to be significantly increased in patients with IBS, even though MC abundance only correlated weakly with IBS symptoms, and, in some cases, a decreased density of colonic c-kit-positive in IgE- or IgG-stained MC, was reported [2], even though $\mathrm{IgE}$ - or IgG-staining is not an index of MC activation.

In the paper by Choi et al. [3] published in this issue of Digestive Diseases and Sciences, the authors analyzed intestinal MC density in: (a) diverse patient cohorts, including diarrhea predominant-IBS (D-IBS), ulcerative colitis (UC) in remission, mild UC, and in healthy controls; (b) random colonic biopsies; (c) different colonic segments; and (d) mucosal MC and lamina propria/intra-epithelial $\mathrm{T}$ lymphocytes. The results are important because MC number was significantly increased in $97.6 \%$ of D-IBS patients, intra-epithelial lymphocytes (IEL) were increased in $92.8 \%$, and lamina propria lymphocytes (LPL) were increased in $81.9 \%$. Moreover, even though the numbers of immune cells were even higher in some post-infectious (PI)-IBS patients, the remaining D-IBS patients had a significantly higher number of these cells compared with controls. Immunochemically identified were tryptase-positive MC and CD3-positive T cells (and not for all lymphocytes, as the authors stated).

All publications to date, including the one by Choi et al. [3], did not report MC activation, which, according to some, is more important than actual numbers [4].

For instance, tryptase staining underestimates the number of degranulated MC, since released secretory granule tryptase does not stain. One way to identify degranulated MC is to stain for c-kit (CD117), the surface MC receptor for stem cell factor. Nevertheless, since mediator secretion can also occur without degranulation [5], MC activation can best be identified either by ultrastructural appearance 
or by qPCR measurement of histidine decarboxylase or tryptase gene expression.

Unfortunately, patients with IBS or UC have not been evaluated for potential triggers, especially their stress status, since stress induces numerous pathological alterations [6], including MC-dependent increased intestinal permeability [7]. A brief period of restraint stress activated mucosal MC and disrupted the gut-blood barrier, as evidenced by breakdown of tight junctions in enteric villi [8]. Corticotropin-releasing hormone $(\mathrm{CRH})$, secreted from the paraventricular nucleus of the hypothalamus in response to stress, activates the hypothalamic-pituitary adrenal (HPA) axis leading to secretion of cortisone that generally has anti-inflammatory effects. Instead, CRH secreted outside the CNS has pro-inflammatory actions [9], apparently through activation of MC [10]. For instance, CRH was reported to increase mucosal barrier permeability, dependent on MC activation [11].

The trigger(s) for MC proliferation and activation may be different in IBS than it is in UC. Stress is important in IBS as discussed above, whereas stress and infections may be more important in UC pathogenesis. For instance, chronic stress disrupted hindgut barrier function, inducing MC-dependent bacterial adhesion and intestinal inflammation [12].

\section{Summary and Future Directions}

MC appear to be increased in IBS $>$ (PI)-IBS $>\mathrm{UC}$ as compared to controls. This finding alone suggest that there is some common underlying pathogenesis involving $\mathrm{MC}$ that may depend on the degree of GI inflammation present. Unfortunately, it is hard to sample the entire gut and use the same preservation and staining techniques to make this approach useful diagnostically. Future studies should focus on GI-MC function by investigating gene expression of unique MC mediators in whole-mount random biopsies or in laser-captured mucosal MC. Given sufficient biopsy tissues, such mediators could also be quantitated using Western blot analysis. Patients should be better characterized and phenotyped for the presence of other related diseases, such as chronic fatigue syndrome, fibromyalgia, mastocytosis, bladder pain syndrome/interstitial cystitis, who share symptoms and other attributes with IBS [13], and may contribute their own pathological characteristics.

Inhibition of mucosal MC may be an effective therapeutic approach since they can also super-stimulate activated T cells [14]. Use of select natural flavonoids, such as luteolin, hold promise for further development since they can inhibit MC and T cell function [14].

\section{References}

1. Rijnierse A, Koster AS, Nijkamp FP, Kraneveld AD. TNF-alpha is crucial for the development of mast cell-dependent colitis in mice. Am J Physiol Gastrointest Liver Physiol. 2006;291:G969G976.

2. Braak B, Klooker TK, Wouters MM, et al. Mucosal immune cell numbers and visceral sensitivity in patients with irritable bowel syndrome: is there any relationship? Am J Gastroenterol. 2012;107:715-726.

3. Ahn YJ, Lee $\mathrm{KH}$, Choi $\mathrm{CH}$, et al. Colonic mucosal immune activity in irritable bowel syndrome: comparison with healthy controls and patients with ulcerative colitis. Dig Dis Sci. 2013. (Epub ahead of print). doi:10.1007/s10620-013-2930-4.

4. Theoharides TC, Asadi S, Chen J, Huizinga JD. Irritable bowel syndrome and the elusive mast cells. Am J Gastroenterol. 2012;107:727-729.

5. Theoharides TC, Kempuraj D, Tagen M, Conti P, Kalogeromitros D. Differential release of mast cell mediators and the pathogenesis of inflammation. Immunol Rev. 2007;217:65-78.

6. Kiank C, Tache Y, Larauche M. Stress-related modulation of inflammation in experimental models of bowel disease and postinfectious irritable bowel syndrome: role of corticotropinreleasing factor receptors. Brain Behav Immun. 2010;24:41-48.

7. Santos J, Benjamin M, Yang PC, Prior T, Perdue MH. Chronic stress impairs rat growth and jejunal epithelial barrier function: role of mast cells. Am J Physiol Gastrointest Liver Physiol. 2000;278:G847-G854.

8. Theoharides TC, Letourneau R, Patra P, et al. Stress-induced rat intestinal mast cell intragranular activation and inhibitory effect of sulfated proteoglycans. Dig Dis Sci. 1999;44:87S-93S.

9. Slominski AT, Zmijewski MA, Zbytek B, Tobin DJ, Theoharides TC, Rivier J. Key Role of CRF in the Skin Stress Response System. Endocr Rev. 2013;34:827-884.

10. Theoharides TC, Donelan JM, Papadopoulou N, Cao J, Kempuraj $\mathrm{D}$, Conti P. Mast cells as targets of corticotropin-releasing factor and related peptides. Trends Pharmacol Sci. 2004;25:563-568.

11. Vanuytsel T, van Wanrooy S, Vanheel H, et al. Psychological stress and corticotropin-releasing hormone increase intestinal permeability in humans by a mast cell-dependent mechanism. Gut. 2013. (Epub ahead of print). doi:10.1136/gutjnl-2013-305690.

12. Soderholm JD, Yang PC, Ceponis P, et al. Chronic stress induces mast cell-dependent bacterial adherence and initiates mucosal inflammation in rat intestine. Gastroenterology. 2002;123: 1099-1108.

13. Theoharides TC. Atopic conditions in search of pathogenesis and therapy. Clin Ther. 2013;35:544-547.

14. Kempuraj D, Tagen M, Iliopoulou BP, et al. Luteolin inhibits myelin basic protein-induced human mast cell activation and mast cell dependent stimulation of Jurkat T cells. $\mathrm{Br} J$ Pharmacol. 2008;155:1076-1084. 